\title{
SUPPORTING INFORMATION FOR: PLATINUM-CATALYZED TANDEM DIBORATION/ASYMMETRIC ALLYLBORATION: ACCESS TO NONRACEMIC FUNCTIONALIZED 1,3-DIOLS.
}

\author{
Jeremy B. Morgan and James P. Morken* \\ Department of Chemistry, Venable and Kenan Laboratories \\ University of North Carolina, Chapel Hill, NC 27599-3290
}

General. ${ }^{1} \mathrm{H}$ NMR spectra were recorded on Bruker DRX (400 or $300 \mathrm{MHz}$ ) spectrometers. Chemical shifts are reported in ppm from tetramethylsilane with the solvent resonance as the internal standard $\left(\mathrm{CDCl}_{3}: 7.24 \mathrm{ppm}\right)$. Data are reported as follows: chemical shift, integration, multiplicity $(\mathrm{s}=$ singlet, $\mathrm{d}=$ doublet, $\mathrm{t}=$ triplet, $\mathrm{q}=$ quartet, $\mathrm{br}=$ broad, $\mathrm{m}=$ multiplet $)$, coupling constants $(\mathrm{Hz})$ and assignment. ${ }^{13} \mathrm{C}$ NMR were recorded on a Bruker $400 \mathrm{MHz}(100 \mathrm{MHz})$ spectrometer with complete proton decoupling. Chemical shifts are reported in ppm from tetramethylsilane with the solvent as the internal standard $\left(\mathrm{CDCl}_{3}: 77.0 \mathrm{ppm}\right)$. Infrared (IR) spectra were obtained using a Nicolet 560 infrared spectrometer.

Liquid chromatography was performed using forced flow (flash chromatography) on silica gel $\left(\mathrm{SiO}_{2}\right.$, 32 to $63 \mu \mathrm{m}$ ) purchased from Scientific Absorbent, Inc. Thin layer chromatography (TLC) was performed on EM science $0.25 \mathrm{~mm}$ silica gel 60 plates. Visualization was achieved with phosphomolybdic acid in ethanol or potassium permanganate in water, each followed by heating.

All reactions were conducted in oven and flame dried glassware under an inert atmosphere of dry nitrogen. ${ }^{1} \mathrm{H}$ NMR samples were prepared in a glovebox under an inert atmosphere of dry nitrogen. Deuterated solvents were used as received. Benzene was distilled over $\mathrm{CaH}_{2}$ and freeze-pump-thaw degassed. Toluene was passed through activated basic alumina. $\mathrm{Pt}(\mathrm{dba})_{2}$ was synthesized by literature procedure (Cherwinski, W. J.; Johnson B.; Lewis, J. J. Chem. Soc., Dalton Trans. 1974, 1404-1409). Aldehydes were purchased from Aldrich and used after distillation. (R)-2,2-Dimethyl-1,3-dioxolane-4carboxaldehyde was synthesized from 1,2:5,6-Di-O-isopropylidene-D-mannitol and (S)-2,2-Dimethyl-1,3dioxolane-4-carboxaldehyde was synthesized from (R)-3-buten-1,2-diol. All other reagents were purchased from Aldrich Chemical Companies and used directly.

Representative Procedure for the Tandem Diboration/Allylation. Using a dry-box under an inert $\mathrm{N}_{2}$ atmosphere, $3.3 \mathrm{mg}(0.005 \mathrm{mmol})$ of $\mathrm{Pt}(\mathrm{dba})_{2}$ and $1.4 \mathrm{mg}(0.005 \mathrm{mmol})$ of tricyclohexyl phosphine were weighed into a dry $25 \mathrm{ml}$ round bottom flask containing a Teflon-coated magnetic stir bar. $95 \mathrm{mg}$ $(0.2 \mathrm{mmol})$ of $(\mathrm{L})$-Bis(diethyltartrateglycato) diboron (90\%) were added to the flask. Upon the addition of $1 \mathrm{~mL}$ of benzene, a purple solution was formed. After stirring momentarily, $16.4 \mathrm{mg}(0.2 \mathrm{mmol})$ of 2,3dimethyl-1,3-butadiene was added to the solution dropwise. The flask was sealed with a rubber septum and the solution was stirred for 12 hours under an inert atmosphere. To the resulting light brown solution was added $80 \mathrm{mg}$ of activated $4 \AA$ molecular sieves. Upon dilution with $2.75 \mathrm{~mL}$ of toluene, the solution was cooled to $-78^{\circ} \mathrm{C}$ in a dry ice/acetone bath. $0.2 \mathrm{mmol}$ of cyclohexane carboxaldehyde $(0.8 \mathrm{M}$ in 
toluene) were added dropwise via syringe over a 45 minute period to ensure that reaction temperature remained at $-78^{\circ} \mathrm{C}$. The reaction mixture was stirred for 3 hours before warming to room temperature. Volatiles were removed in vacuo leaving a brown residue, which was subsequently dissolved in $4 \mathrm{~mL}$ of THF. $907 \mu \mathrm{L}(8 \mathrm{mmol})$ of $30 \% \mathrm{H}_{2} \mathrm{O}_{2}$ and $800 \mu \mathrm{L}(2.4 \mathrm{mmol})$ of $3 \mathrm{M} \mathrm{NaOH}$ were added. The two-phase mixture was heated to $50^{\circ} \mathrm{C}$ for 2 hours. The reaction was quenched with $5 \mathrm{~mL}$ of saturated sodium thiosulfate, and the aqueous layer was extracted with $2 \times 20 \mathrm{~mL}$ of ethyl acetate. The combined organic layers were dried with $\mathrm{Na}_{2} \mathrm{SO}_{4}$, filtered and concentrated. The crude material was purified by column chromatography (silica gel, 3:1 hexane:ethyl acetate). Isolated yield of $30.6 \mathrm{mg}(0.14 \mathrm{mmol}, 72 \%$ yield).

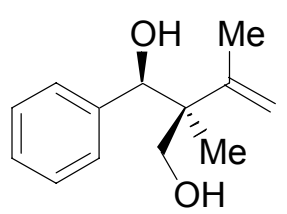

(1R,2S)-2-Isopropenyl-2-methyl-1-phenyl-propane-1,3-diol. ${ }^{1} \mathrm{H}$ NMR (400 $\mathrm{MHz}$, $\left.\mathrm{CDCl}_{3}\right): 7.22-7.31(5 \mathrm{H}$, br m, aryl CH$), 5.01(1 \mathrm{H}, \mathrm{m}, \mathrm{C}=\mathrm{CH}), 4.93(1 \mathrm{H}, \mathrm{s}, \mathrm{C}=\mathrm{CH}), 4.87$ $(1 \mathrm{H}, \mathrm{s}, \mathrm{CHOH}), 3.75\left(1 \mathrm{H}, \mathrm{d},{ }^{2} \mathrm{~J}_{\mathrm{AB}}=11.2 \mathrm{~Hz}, \mathrm{CH}_{2} \mathrm{OH}\right), 3.60\left(1 \mathrm{H}, \mathrm{d},{ }^{2} \mathrm{~J}_{\mathrm{AB}}=11.2 \mathrm{~Hz}\right.$, $\left.\mathrm{CH}_{2} \mathrm{OH}\right), 1.73\left(3 \mathrm{H}, \mathrm{s}, \mathrm{C}=\mathrm{CCH}_{3}\right), 0.85\left(3 \mathrm{H}, \mathrm{s}, \mathrm{CCH}_{3}\right) .{ }^{13} \mathrm{C}$ NMR (100 MHz): 147.1, 140.7, 127.6, 127.5, 127.4, 113.2, 79.4, 67.9, 48.1, 20.6, 17.6. IR: 3460 (br), 3054 (m), 2987 (m), 1639 (m), 1422 (m), 1265 (s). HRMS Calc'd for $\mathrm{C}_{13} \mathrm{H}_{18} \mathrm{O}_{2} \mathrm{M}+\mathrm{NH}_{4}$ : 224.1651; Found: 224.1660 .

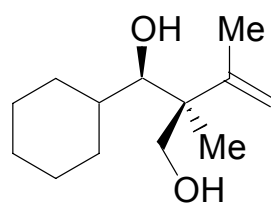

(1R,2S)-1-Cyclohexyl-2-isopropenyl-2-methyl-propane-1,3-diol. ${ }^{1} \mathrm{H} \quad \mathrm{NMR} \quad(400$ $\left.\mathrm{MHz}, \mathrm{CDCl}_{3}\right): 4.97(1 \mathrm{H}, \mathrm{m}, \mathrm{C}=\mathrm{CH}), 4.89(1 \mathrm{H}, \mathrm{s}, \mathrm{C}=\mathrm{CH}), 3.67(1 \mathrm{H}, \mathrm{d}, \mathrm{J}=4.0 \mathrm{~Hz}$, $\mathrm{CHOH}), 3.66\left(1 \mathrm{H}, \mathrm{d},{ }^{2} \mathrm{~J}_{\mathrm{AB}}=10.8 \mathrm{~Hz}, \mathrm{CH}_{2} \mathrm{OH}\right), 3.50\left(1 \mathrm{H}, \mathrm{d},{ }^{2} \mathrm{~J}_{\mathrm{AB}}=10.8 \mathrm{~Hz}, \mathrm{CH}_{2} \mathrm{OH}\right), 1.74$ $\left(3 \mathrm{H}, \mathrm{d},{ }^{4} \mathrm{~J}=0.8 \mathrm{~Hz}, \mathrm{C}=\mathrm{CCH}_{3}\right), 1.4-1.9(5 \mathrm{H}, \mathrm{m}$, ring $\mathrm{CH}), 1.1(6 \mathrm{H}$, br m, ring $\mathrm{CH}), 1.06$ $\left(3 \mathrm{H}, \mathrm{s}, \mathrm{CCH}_{3}\right) .{ }^{13} \mathrm{C} \mathrm{NMR}(100 \mathrm{MHz}): 147.7,113.1,79.7,69.8,48.8,40.2,32.9,28.3$, 26.7, 26.3, 26.2, 20.5, 15.6. IR: 3444 (br), 3054 (m), 2987 (m), 1636 (m), 1422 (m), 1265 (s). HRMS Calc'd for $\mathrm{C}_{13} \mathrm{H}_{24} \mathrm{O}_{2} \mathrm{M}+\mathrm{NH}_{4}$ : 230.2120; Found: 230.2124 .

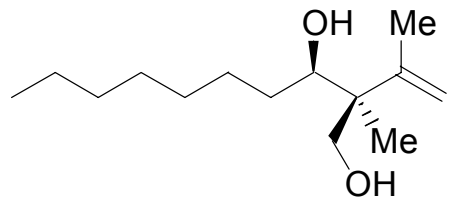

(1R,2S)-2-Isopropenyl-2-methyl-decane-1,3-diol. ${ }^{1} \mathrm{H}$ NMR (400 MHz, $\left.\mathrm{CDCl}_{3}\right): 4.98(1 \mathrm{H}, \mathrm{m}, \mathrm{C}=\mathrm{CH}), 4.89(1 \mathrm{H}, \mathrm{s}, \mathrm{C}=\mathrm{CH}), 3.80(1 \mathrm{H}, \mathrm{dd}, \mathrm{J}=9.6 \mathrm{~Hz}$, $\mathrm{J}=2.0 \mathrm{~Hz} \mathrm{CHOH}), 3.71\left(1 \mathrm{H}, \mathrm{d},{ }^{3} \mathrm{~J}_{\mathrm{AB}}=10.8 \mathrm{~Hz}, \mathrm{CH}_{2} \mathrm{OH}\right), 3.57(1 \mathrm{H}, \mathrm{d}$, $\left.{ }^{3} \mathrm{~J}_{\mathrm{AB}}=10.8 \mathrm{~Hz}, \mathrm{CH} \mathbf{H}_{2} \mathrm{OH}\right), 1.72\left(3 \mathrm{H}, \mathrm{d},{ }^{4} \mathrm{~J}=0.8 \mathrm{~Hz}, \mathrm{C}=\mathrm{CCH}_{3}\right), 1.1-1.6(13 \mathrm{H}, \mathrm{m}$, aliphatic $\mathrm{CH}), 0.99\left(3 \mathrm{H}, \mathrm{s}, \mathrm{CCH}_{3}\right), 0.85\left(3 \mathrm{H}, \mathrm{t}, \mathrm{J}=6.8 \mathrm{~Hz}, \mathrm{CH}_{2} \mathrm{CH}_{3}\right) .{ }^{13} \mathrm{C}$ NMR (100 MHz): 147.4, 113.1, 76.0, 69.0, 48.1, 31.9, 31.8, 29.6, 29.3, 26.9, 22.6, 20.2, 15.3, 14.4. IR: 3427 (br), 3054 (m), 2928 (m), 2856 (m), 1635 (m), 1422 (m), 1265 (s). HRMS Calc'd for $\mathrm{C}_{14} \mathrm{H}_{28} \mathrm{O}_{2}$ $\mathrm{M}+\mathrm{NH}_{4}$ : 246.2433; Found: 246.2442.

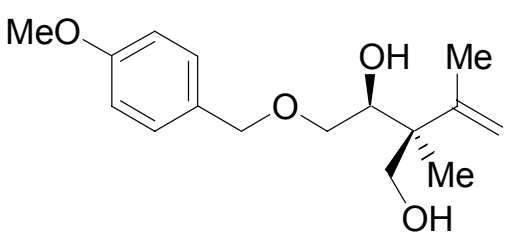

(1S,2S)-2-Isopropenyl-4-(4-methoxy-benzyloxy)-2-methyl-butane-1,3diol. ${ }^{1} \mathrm{H}$ NMR (400 MHz, $\left.\mathrm{CDCl}_{3}\right): 7.23(2 \mathrm{H}, \mathrm{d}, \mathrm{J}=8.4 \mathrm{~Hz}$, aromatic $\mathrm{CH})$, $6.86(2 \mathrm{H}, \mathrm{d}, \mathrm{J}=8.4 \mathrm{~Hz}$, aromatic $\mathrm{CH}), 4.95(1 \mathrm{H}, \mathrm{m}, \mathrm{C}=\mathrm{CH}), 4.89(1 \mathrm{H}, \mathrm{s}$, $\mathrm{C}=\mathrm{CH}), 4.44\left(2 \mathrm{H}, \mathrm{s}, \mathrm{CCH}_{2} \mathrm{O}\right), 4.08(1 \mathrm{H}, \mathrm{dd}, \mathrm{J}=8.4 \mathrm{~Hz}, \mathrm{~J}=2.4 \mathrm{~Hz}, \mathrm{CHOH})$, $3.79\left(1 \mathrm{H}, \mathrm{s}, \mathrm{OCH}_{3}\right), 3.74\left(1 \mathrm{H}, \mathrm{d},{ }^{2} \mathrm{~J}_{\mathrm{AB}}=11.2 \mathrm{~Hz}, \mathrm{CH}_{2} \mathrm{OH}\right), 3.50(1 \mathrm{H}, \mathrm{d}$, $\left.{ }^{2} \mathrm{~J}_{\mathrm{AB}}=11.2 \mathrm{~Hz}, \mathrm{CH}_{2} \mathrm{OH}\right), 3.47\left(1 \mathrm{H}, \mathrm{dd}, \mathrm{J}=9.6 \mathrm{~Hz}, \mathrm{~J}=2.4 \mathrm{~Hz}, \mathrm{OCH}_{2} \mathrm{CHOH}\right), 3.37(1 \mathrm{H}, \mathrm{dd}, \mathrm{J}=8.4 \mathrm{~Hz}, \mathrm{~J}=9.6$ $\left.\mathrm{Hz}, \mathrm{OCH} \mathbf{H}_{2} \mathrm{CHOH}\right), 1.72\left(3 \mathrm{H}, \mathrm{s}, \mathrm{C}=\mathrm{CCH}_{3}\right), 1.04\left(3 \mathrm{H}, \mathrm{s}, \mathrm{CCH}_{3}\right) .{ }^{13} \mathrm{C}$ NMR (100 MHz): 159.3, 146.4, 
129.8, 129.3, 113.9, 113.4, 74.2, 73.1, 70.9, 69.1, 55.3, 46.3, 20.1, 15.2. IR: 3421 (br), 3055 (m), 2987 (m), 1639 (m), 1422 (m), 1265 (s). HRMS Calc'd for $\mathrm{C}_{16} \mathrm{H}_{24} \mathrm{O}_{4} \mathrm{M}+\mathrm{NH}_{4}$ : 298.2018; Found: 298.2013.

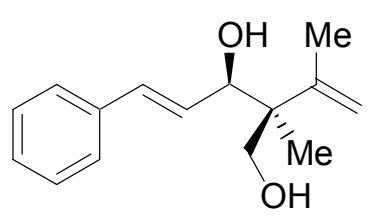

(1R,2S)-2-Isopropenyl-2-methyl-5-phenyl-pent-4-ene-1,3-diol. ${ }^{1} \mathrm{H}$ NMR (400 $\left.\mathrm{MHz}, \mathrm{CDCl}_{3}\right)$ : 7.2-7.4 (5H, m, ring $\left.\mathrm{CH}\right), 6.64(1 \mathrm{H}, \mathrm{d}, \mathrm{J}=16.0 \mathrm{~Hz}, \mathrm{CH}=\mathrm{CHCHOH})$, $6.27(1 \mathrm{H}, \mathrm{dd}, \mathrm{J}=16.0 \mathrm{~Hz}, \mathrm{~J}=6.0 \mathrm{~Hz}, \mathrm{CH}=\mathrm{CHCHOH}), 5.06\left(1 \mathrm{H}, \mathrm{m}, \mathrm{C}=\mathrm{CH}_{2}\right), 5.01$ $\left(1 \mathrm{H}, \mathrm{s}, \mathrm{C}=\mathrm{CH}_{2}\right), 4.55(1 \mathrm{H}, \mathrm{s}, \mathrm{CH}=\mathrm{CHCHOH}), 3.82\left(1 \mathrm{H}, \mathrm{d},{ }^{2} \mathrm{~J}_{\mathrm{AB}}=11.2 \mathrm{~Hz}, \mathrm{CH}_{2} \mathrm{OH}\right)$, $3.72\left(1 \mathrm{H}, \mathrm{d},{ }^{2} \mathrm{~J}_{\mathrm{AB}}=11.2 \mathrm{~Hz}, \mathrm{CH}_{2} \mathrm{OH}\right), 1.83\left(3 \mathrm{H}, \mathrm{d},{ }^{4} \mathrm{~J}=0.8 \mathrm{~Hz}, \mathrm{C}=\mathrm{CCH}\right), 1.03(3 \mathrm{H}$, s, $\left.\mathrm{CCH}_{3}\right) .{ }^{13} \mathrm{C} \mathrm{NMR}(100 \mathrm{MHz}): 146.9,136.7,131.7,128.6,128.3,127.7,126.5,113.4,76.7,68.3,48.3$, 20.3, 16.9. IR: 3448 (br), 3054 (m), 2987 (m), 1639 (m), 1422 (m), 1265 (s). HRMS Calc'd for $\mathrm{C}_{15} \mathrm{H}_{20} \mathrm{O}_{2}$ $\mathrm{M}+\mathrm{NH}_{4}: 250.1807$; Found: 250.1808 .

(1R,2S)-1-Cyclohexyl-2-isopropenyl-propane-1,3-diol. Catalysis of the diboration

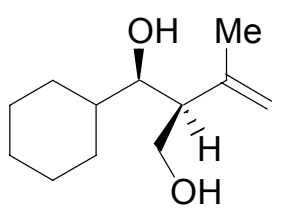

proceeded with $0.01 \mathrm{mmol}$ of ethylenebis(triphenylphosphine)platinum(0). The reaction required heating to $80{ }^{\circ} \mathrm{C}$ for 24 hours to reach completion. The allylation reaction was then carried out as described in the general procedure. ${ }^{1} \mathrm{H}$ NMR $\left(400 \mathrm{MHz}, \mathrm{CDCl}_{3}\right)$ : $4.90(1 \mathrm{H}, \mathrm{m}, \mathrm{C}=\mathrm{CH}), 4.80(1 \mathrm{H}, \mathrm{s}, \mathrm{C}=\mathrm{CH}), 3.84\left(1 \mathrm{H}, \mathrm{dd},{ }^{2} \mathrm{~J}_{\mathrm{AB}}=10.8 \mathrm{~Hz},{ }^{3} \mathrm{~J}_{\mathrm{AX}}=6.8 \mathrm{~Hz}\right.$, $\left.\mathrm{CH}_{2} \mathrm{OH}\right), 3.70\left(1 \mathrm{H}, \mathrm{dd},{ }^{2} \mathrm{~J}_{\mathrm{AB}}=10.8 \mathrm{~Hz},{ }^{3} \mathrm{~J}_{\mathrm{BX}}=5.2 \mathrm{~Hz}, \mathrm{CH}_{2} \mathrm{OH}\right), 3.59(1 \mathrm{H}, \mathrm{dd}, \mathrm{J}=8.8 \mathrm{~Hz}, 3.2 \mathrm{~Hz}, \mathrm{CHOH})$, $2.43\left(1 \mathrm{H}, \mathrm{ddd}, \mathrm{J}=8.8 \mathrm{~Hz}, 6.8 \mathrm{~Hz}, 5.2 \mathrm{~Hz}, \mathrm{CHCH}_{2} \mathrm{OH}\right), 1.69\left(3 \mathrm{H}, \mathrm{s}, \mathrm{C}=\mathrm{CCH}_{3}\right), 1.6-1.8(4 \mathrm{H}, \mathrm{m}$, aliphatic CH), 1.3-1.55 (2H, m, aliphatic CH), 1.0-1.3 (5H, m, aliphatic CH). ${ }^{13} \mathrm{C}$ NMR (100 MHz): 143.7, 113.6, 78.2, 64.9, 51.0, 40.4, 30.4, 26.5, 26.4, 26.1, 25.5, 21.4. IR: 3450 (br), 3054 (m), 2987 (m), 2929 (m), 1644 (m), 1422 (m), 1265 (s). HRMS Calc'd for $\mathrm{C}_{12} \mathrm{H}_{22} \mathrm{O}_{2} \mathrm{M}+\mathrm{NH}_{4}$ : 216.1964; Found: 216.1960.

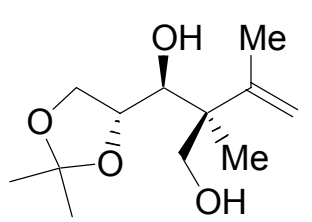

(1S,2S,4'R)-1-(2,2-Dimethyl-[1,3]dioxolan-4-yl)-2-isopropenyl-2-methyl-propane1,3-diol. ${ }^{1} \mathrm{H}$ NMR (400 MHz, $\left.\mathrm{CDCl}_{3}\right): 5.01(1 \mathrm{H}, \mathrm{m}, \mathrm{C}=\mathrm{CH}), 4.91(1 \mathrm{H}, \mathrm{s}, \mathrm{C}=\mathrm{CH}), 4.13$ $(1 \mathrm{H}$, br d, $\mathrm{CHOH}), 4.08\left(1 \mathrm{H}, \mathrm{ddd}, \mathrm{J}=7.2 \mathrm{~Hz}, 7.2 \mathrm{~Hz}, 2.8 \mathrm{~Hz}, \mathrm{CHOC}\left(\mathrm{CH}_{3}\right)_{2}\right), 3.94(1 \mathrm{H}$, $\left.\mathrm{dd}, \mathrm{J}=8.0 \mathrm{~Hz}, 7.2 \mathrm{~Hz}, \mathrm{CH}_{2} \mathrm{OC}\left(\mathrm{CH}_{3}\right)_{2}\right), 3.91\left(1 \mathrm{H}, \mathrm{dd}, \mathrm{J}=8.0 \mathrm{~Hz}, 7.2 \mathrm{~Hz}, \mathrm{CH}_{2} \mathrm{OC}\left(\mathrm{CH}_{3}\right)_{2}\right)$, $3.77\left(1 \mathrm{H}, \mathrm{d},{ }^{2} \mathrm{~J}_{\mathrm{AB}}=11.2 \mathrm{~Hz}, \mathrm{CH}_{2} \mathrm{OH}\right), 3.53\left(1 \mathrm{H}, \mathrm{d},{ }^{2} \mathrm{~J}_{\mathrm{AB}}=11.2 \mathrm{~Hz}, \mathrm{CH}_{2} \mathrm{OH}\right), 1.78(3 \mathrm{H}, \mathrm{d}$, $\left.{ }^{4} \mathrm{~J}=0.8 \mathrm{~Hz}, \mathrm{C}=\mathrm{CCH}_{3}\right), 1.39\left(3 \mathrm{H}, \mathrm{s}, \mathrm{C}\left(\mathrm{CH}_{3}\right)_{2}\right), 1.31\left(3 \mathrm{H}, \mathrm{s}, \mathrm{C}\left(\mathrm{CH}_{3}\right)_{2}\right), 1.01(3 \mathrm{H}, \mathrm{s}, \mathrm{CCH})_{3} .{ }^{13} \mathrm{C} \mathrm{NMR}(100$ $\mathrm{MHz}): 146.5,113.7,107.7,76.5,75.0,69.1,64.5,46.6,26.4,25.1,20.2$, 15.9. IR: 3445 (br), 3055 (m), $2987(\mathrm{~m}), 1636$ (m), 1422 (m), 1266 (s). HRMS Calc'd for $\mathrm{C}_{12} \mathrm{H}_{22} \mathrm{O}_{4} \mathrm{M}+\mathrm{H}$ : 231.1596; Found: 231.1599 .

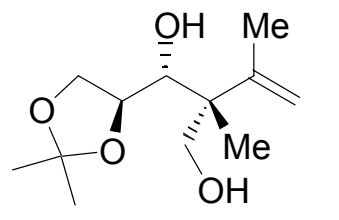

1

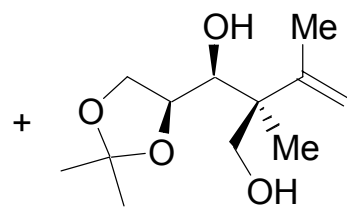

2
1-(2,2-Dimethyl-[1,3]dioxolan-4-yl)-2-isopropenyl-2-methylpropane-1,3-diol. ${ }^{1} \mathrm{H}$ NMR $\left(400 \mathrm{MHz}, \mathrm{CDCl}_{3}\right)$ : An equal mixture of diastereomers was formed one of which was identical to the $1 \mathrm{~S}, 2 \mathrm{~S}, 4^{\prime} \mathrm{R}$ isomer described above. The other was characterized by ${ }^{13} \mathrm{C}$ NMR $(100 \mathrm{MHz})$ only: $146.7,113.9$, 109.6, 74.6, 73.5, 68.7, 67.5, 47.8, 26.2, 25.8, 20.3, 15.8.

HRMS Calc'd for $\mathrm{C}_{12} \mathrm{H}_{22} \mathrm{O}_{4} \mathrm{M}+\mathrm{H}$ : 231.1596; Found: 231.1586 . 
Determination of Enantiopurity. Each diol was converted to the bis-Mosher's Ester via the following procedure: $0.05 \mathrm{mmol}$ of the corresponding diol was transferred to a small vial containing 0.1 mmol of dimethylaminopyridine. $500 \mu \mathrm{L}$ of $\mathrm{CDCl}_{3}$ was added followed by triethylamine $(0.25 \mathrm{mmol})$ and $(R)-(-)$-Mosher's acid chloride $(0.11 \mathrm{mmol}$, Fluka). The yellow solutions were allowed to stand for $1-$ 8 hours, depending on diol structure and then analyzed by ${ }^{1} \mathrm{H}-\mathrm{NMR}$.

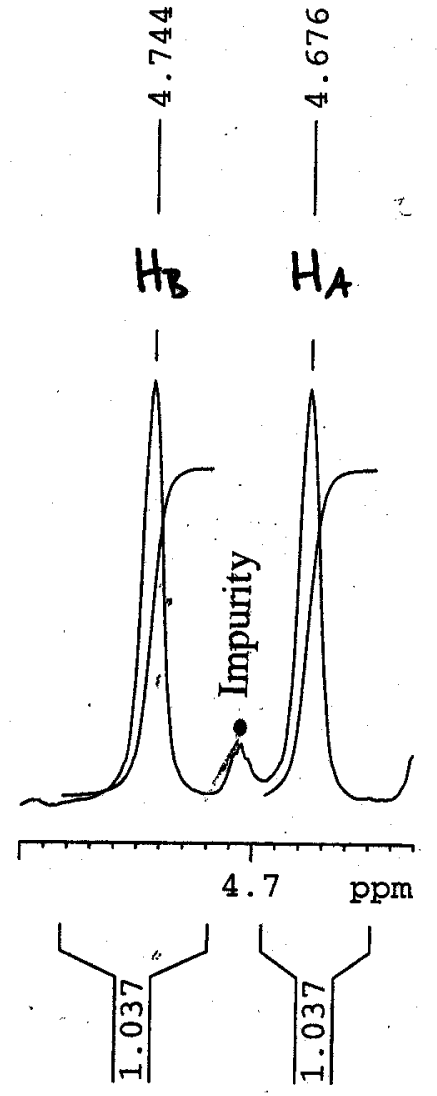

Representative ${ }^{1} \mathrm{H}-\mathrm{NMR}$ peaks for racemic Mosher's ester product
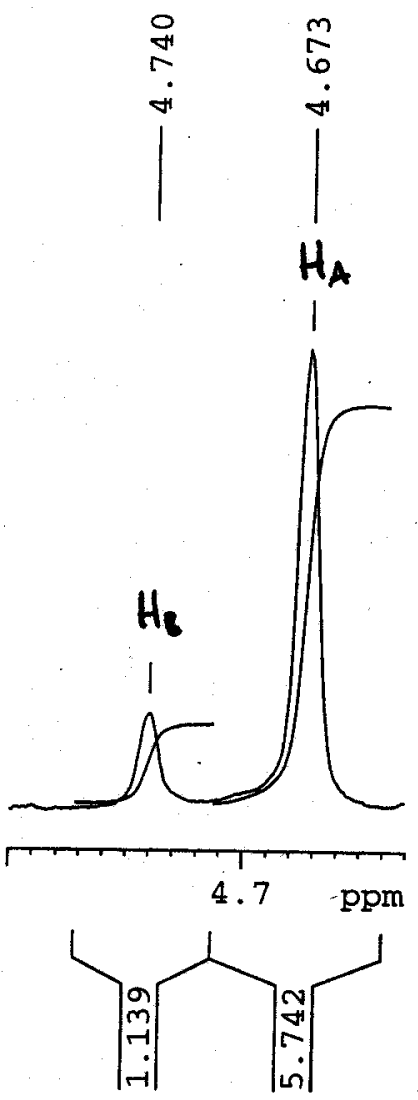

Representative ${ }^{1} \mathrm{H}-\mathrm{NMR}$ peaks for enatioenriched Mosher's ester product

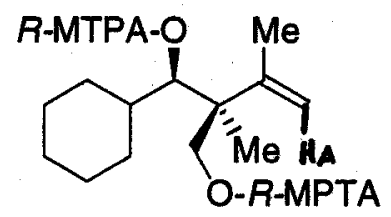

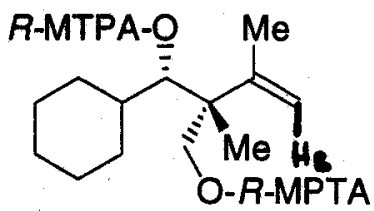

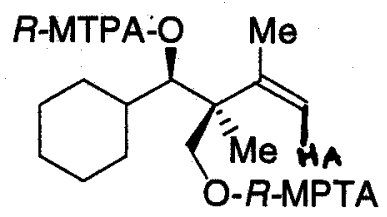

Proof of Absolute Stereochemistry. The absolute configuration of the 1,3-diol products was proven by independent synthesis as shown in the following schemes. In both examples, the absolute 
configuration is derived from the Sharpless asymmetric epoxidation using L-diisopropyl tartrate (Gao, Y.; Hanson, R. M.; Klunder, S.; Masamune, H.; Sharpless, K. B. J. Am. Chem. Soc. 1987, 109, 5765-5780). For compound 3 (Scheme A), the quaternary center is set by a 1,2-isopropenyl shift initiated by Lewis acid induced epoxide opening (Shimazaki, M.; Hara, H.; Suzuki, K.; Tsuchihashi, G. Tetrahedron Lett. 1987, 28, 5891-5894). For compound 4 (Scheme B), the epoxide is opened with iso-propenyl cuprate prepared in situ (Roush, W. R.; Ando, K.; Powers, D. B.; Palkowitz, A. D.; Halterman, R. L. J. Am. Chem. Soc. 1990, 112, 6339-6348). Each diol product was converted to the bis-Mosher's ester as described previously. In both cases, the major resonance corresponded to the minor product formed in the diboration/allylation were present. Based on this observation, the major products formed in the diboration/allylation with L-tartrate-derived bis-boronate are the enantiomer of the independently synthesized diols.

\section{Scheme A}

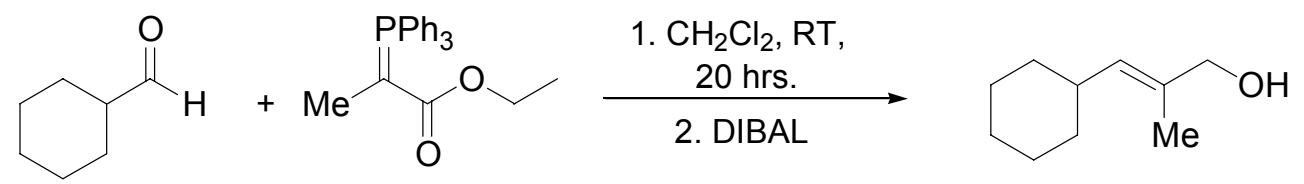

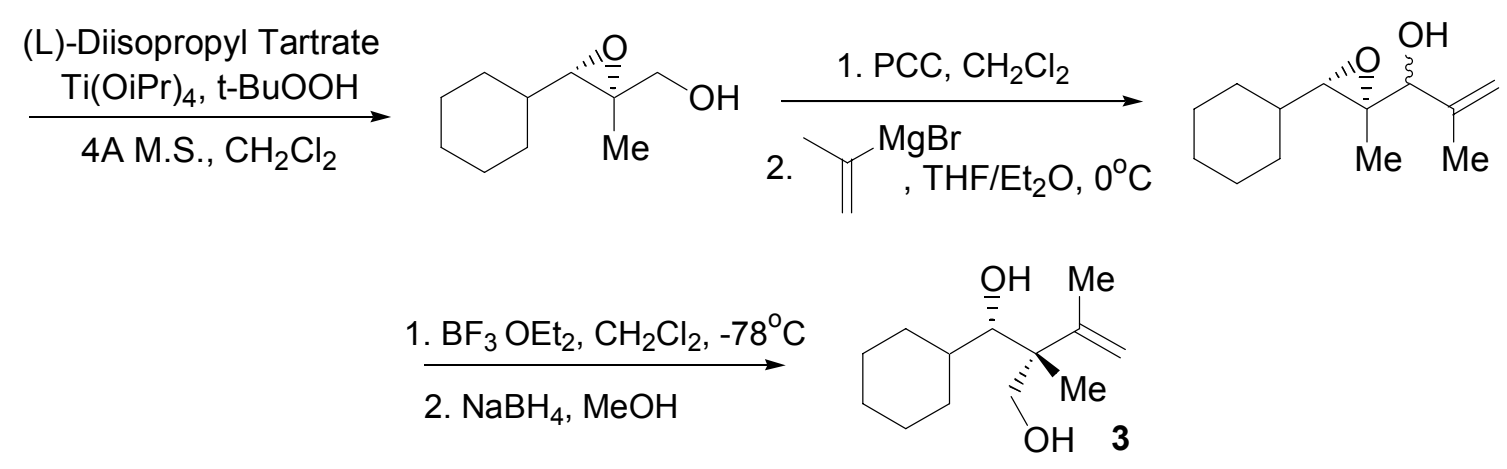

\section{Scheme B}

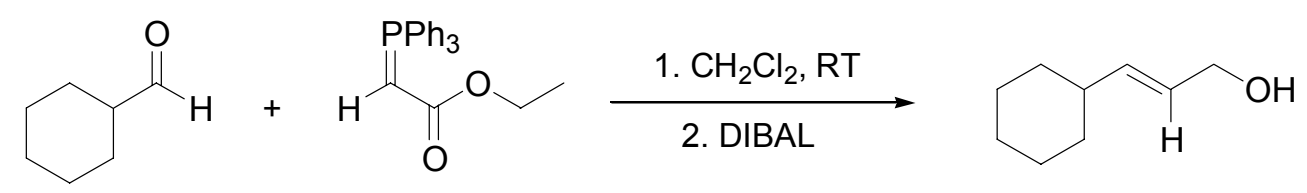

(L)-Diisopropyl Tartrate $\underset{4 \mathrm{~A} \text { M.S., } \mathrm{CH}_{2} \mathrm{Cl}_{2}}{\stackrel{\mathrm{Ti}(\mathrm{OiPr})_{4}, \mathrm{t}-\mathrm{BuOOH}}{\longrightarrow}}$

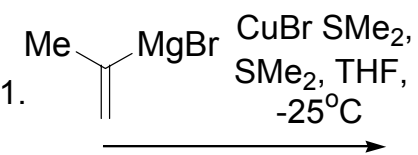

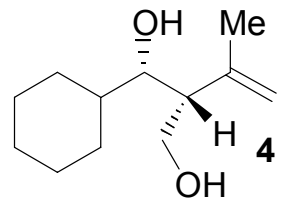




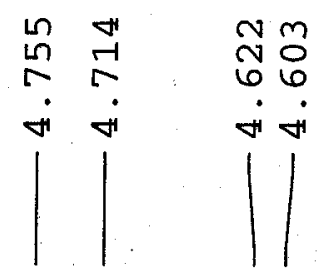

vinylic C-H's

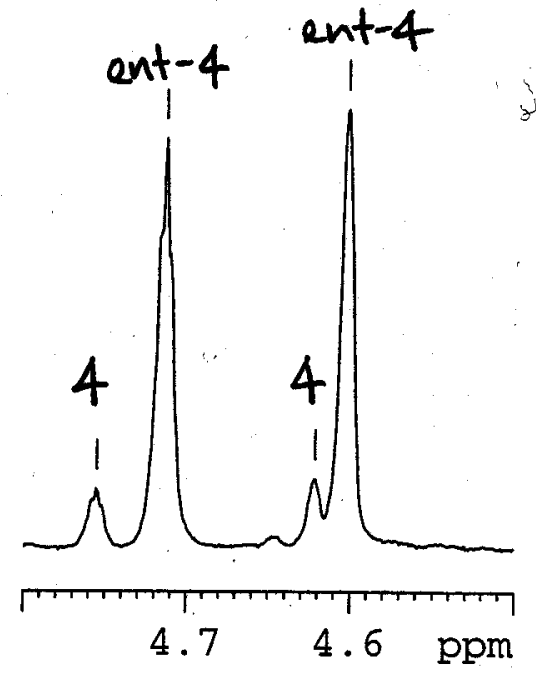

Mosher's ester of diol 4 from diboration/allylation.

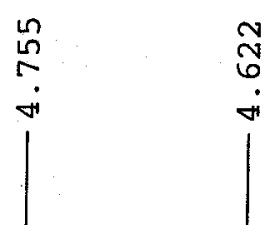

vinylic $\mathrm{C}-\mathrm{H}_{\mathrm{s}}$

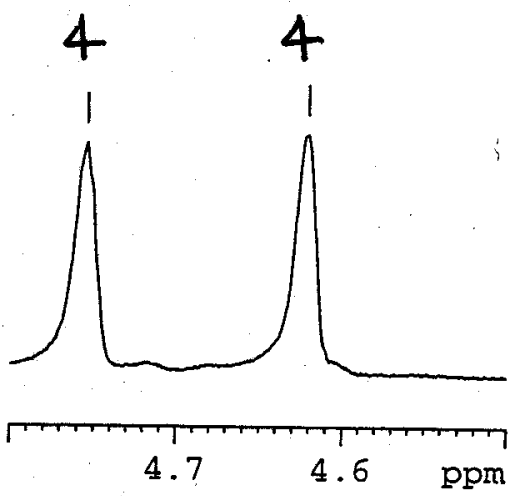

Mosher's ester of diol 4 from authentic synthesis (Scheme B) 
Procedure for Cyclic Boronate Formation. The general procedure was followed for diboration and reaction with aldehyde. Solvent was removed in vacuo, and the crude product was re-dissolved in THF $(0.2 \mathrm{M}) . \quad 0.5 \mathrm{mmol}$ of dimethyl zinc ( $2 \mathrm{M}$ in toluene) was added dropwise to the stirred solution at room temperature. After 2 hours of stirring, the solution was cooled to $0^{\circ} \mathrm{C}$ and quenched with $1 \mathrm{~mL}$ of water. $10 \mathrm{~mL}$ of ethyl acetate was added. After removal of the organic layer, the aqueous layer was extracted with $2 \times 20 \mathrm{~mL}$ of ethyl acetate. The combined organic layers were dried with $\mathrm{Na}_{2} \mathrm{SO}_{4}$, filtered and concentrated. The crude material was purified by column chromatography (silica gel, 10:1 hexane:ethyl acetate). Isolated yield of $28.5 \mathrm{mg}(0.12 \mathrm{mmol}, 60 \%)$.

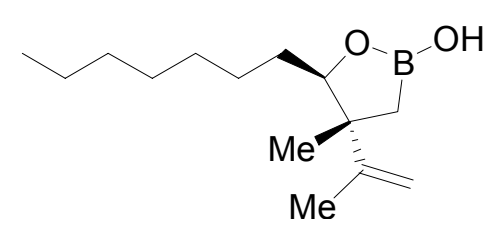

(4S,5R)-5-heptyl-4-methyl-4-(2-propenyl)-[1,2]-oxoborolane-2-ol. $\quad{ }^{1} \mathrm{H}$ NMR (400 MHz, $\left.\mathrm{CDCl}_{3}\right): 4.75(1 \mathrm{H}$, br s, C=CH), $4.72(1 \mathrm{H}$, br s, OH), 4.68 $(1 \mathrm{H}, \mathrm{s}, \mathrm{C}=\mathrm{CH}), 4.11\left(1 \mathrm{H}, \mathrm{dd},{ }^{3} \mathrm{~J}_{\mathrm{AB}}=8.4 \mathrm{~Hz},{ }^{3} \mathrm{~J}_{\mathrm{AX}}=6.4 \mathrm{~Hz} \mathrm{CHOH}\right), 1.76(1 \mathrm{H}$, $\left.\mathrm{s}, \mathrm{C}=\mathrm{CCH}_{3}\right), 1.53\left(2 \mathrm{H}, \mathrm{m}, \mathrm{CH}_{2} \mathrm{CHOB}\right), 1.35-1.41\left(2 \mathrm{H}, \mathrm{m}, \mathrm{CH}_{2} \mathrm{~B}\right), 1.1-1.35$ $(10 \mathrm{H}, \mathrm{m}$, chain $\mathrm{CH}), 0.99\left(3 \mathrm{H}, \mathrm{s}, \mathrm{CCH}_{3}\right), 0.87\left(3 \mathrm{H}, \mathrm{t},{ }^{3} \mathrm{~J}_{\mathrm{CH}}=5.6 \mathrm{~Hz}\right.$, $\left.\mathrm{CH}_{2} \mathrm{CH}_{3}\right) .{ }^{13} \mathrm{C}$ NMR $(100 \mathrm{MHz}): 150.1,109.7,84.8,48.9,31.8,31.6,31.2,29.6,29.2,27.3,22.6,20.5$, 20.3, 14.1. LRMS Calc'd for $\mathrm{C}_{14} \mathrm{H}_{27} \mathrm{BO}_{2} \mathrm{M}+\mathrm{HOCH}_{3}$ : 270.2; Found: 270.3 .

Procedure for Homologation to 1,4-diol. Boronate $(0.05 \mathrm{mmol})$ was transferred as a solution in THF $(0.15 \mathrm{M})$ to a $10 \mathrm{ml}$ round bottom flask containing a stir bar. $0.10 \mathrm{mmol}$ of bromochloromethane was added and the solution was cooled to $-78^{\circ} \mathrm{C}$ in a dry ice/acetone bath. Butyllithium (1.55 $\mathrm{M}$ in hexanes) was added dropwise while the solution was stirred. After 5 minutes, the solution was warmed to room temperature overnight. $250 \mu \mathrm{L}$ of $30 \% \mathrm{H}_{2} \mathrm{O}_{2}$ and $200 \mu \mathrm{L}$ of $3 \mathrm{M} \mathrm{NaOH}$ were added. The two-phase mixture was heated to $50^{\circ} \mathrm{C}$ for 2 hours. The reaction was quenched with $2 \mathrm{~mL}$ of sodium thiosulfate, and $10 \mathrm{~mL}$ was ethyl acetate was added. After removal of the organic layer, the aqueous layer was extracted with $2 \times 20 \mathrm{~mL}$ of ethyl acetate. The combined organic layers were dried with $\mathrm{Na}_{2} \mathrm{SO}_{4}$, filtered and concentrated. The crude material was purified by column chromatography (silica gel, 3:1 hexane:ethyl acetate). Isolated yield of $4.4 \mathrm{mg}(0.02 \mathrm{mmol}, 36 \%)$.

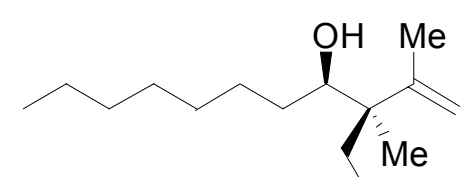

$\mathrm{HO}$

(3R, 4R)-3-Isopropenyl-3-methyl-undecane-1,4-diol. ${ }^{1} \mathrm{H}$ NMR (400 MHz, $\left.\mathrm{CDCl}_{3}\right): 4.88(1 \mathrm{H}, \mathrm{m}, \mathrm{C}=\mathrm{CH}), 4.78(1 \mathrm{H}, \mathrm{s}, \mathrm{C}=\mathrm{CH}), 3.62\left(2 \mathrm{H}, \mathrm{t},{ }^{3} \mathrm{~J}_{\mathrm{CH}}=6.4 \mathrm{~Hz}\right.$, $\left.\mathrm{CH}_{2} \mathrm{OH}\right), 3.58\left(1 \mathrm{H}\right.$, br $\left.\mathrm{d},{ }^{3} \mathrm{~J}_{\mathrm{AB}}=10.0 \mathrm{~Hz}, \mathrm{CHOH}\right), 1.70-1.85$ (2H, m, $\left.\mathrm{CCH}_{2} \mathrm{CH}_{2} \mathrm{OH}\right), 1.69\left(3 \mathrm{H}, \mathrm{s}, \mathrm{C}=\mathrm{CCH}_{3}\right), 1.4-1.6\left(2 \mathrm{H}, \mathrm{m}, \mathrm{CH}_{2} \mathrm{CHOH}\right), 1.1-1.4$ $(10 \mathrm{H}, \mathrm{m}$, chain $\mathrm{CH}), 0.98\left(3 \mathrm{H}, \mathrm{s}, \mathrm{CCH}_{3}\right), 0.85\left(3 \mathrm{H}, \mathrm{t},{ }^{3} \mathrm{~J}_{\mathrm{CH}}=6.8 \mathrm{~Hz}, \mathrm{CH}_{2} \mathrm{CH}_{3}\right)$. ${ }^{13} \mathrm{C}$ NMR (100 MHz): 149.4, 112.2, 76.0, 59.7, 46.2, 39.3, 31.8, 31.4, 29.6, 29.3, 27.2, 22.6, 20.0, 17.2, 14.1. . LRMS Calc'd for $\mathrm{C}_{15} \mathrm{H}_{30} \mathrm{O}_{2} \mathrm{M}-\mathrm{H}_{2} \mathrm{O}$ : 225.2; Found: 225.3 . 


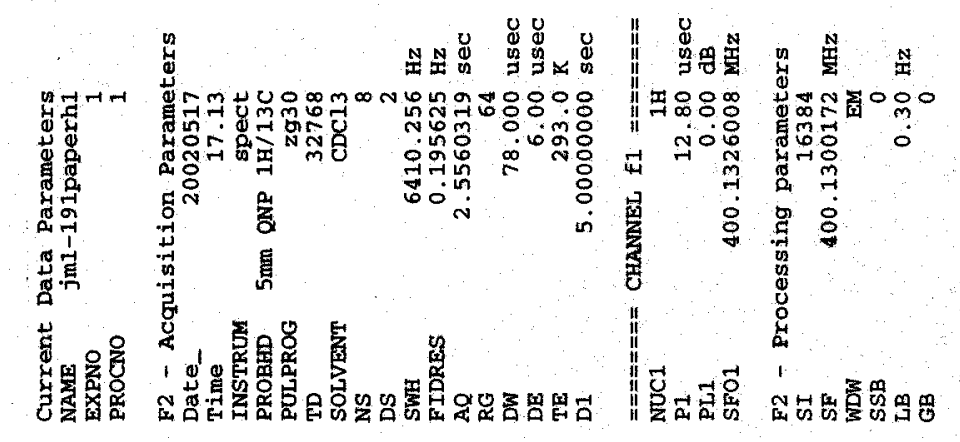

$\varepsilon \subseteq 8^{\circ} 0$
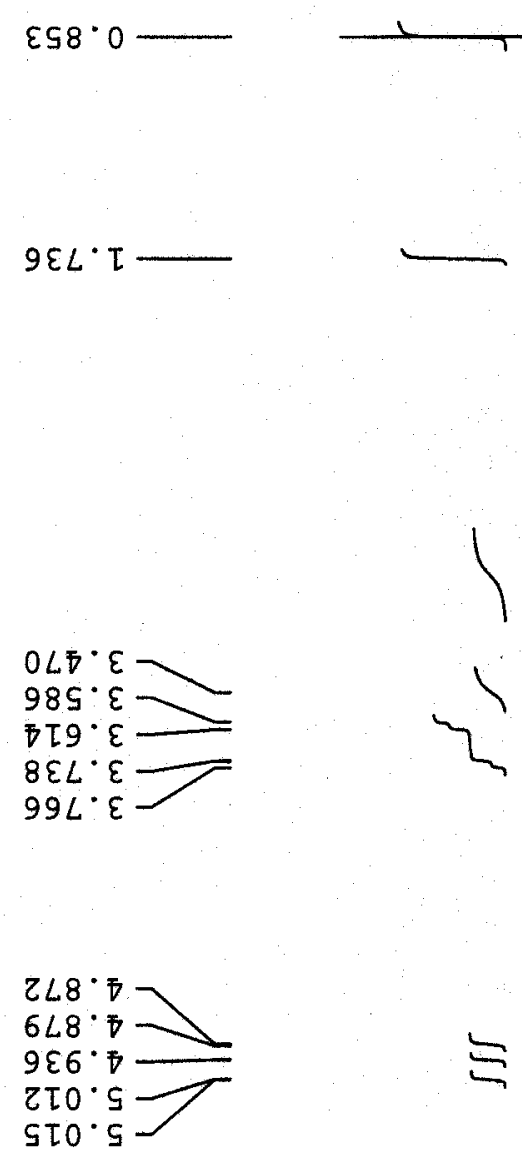

5
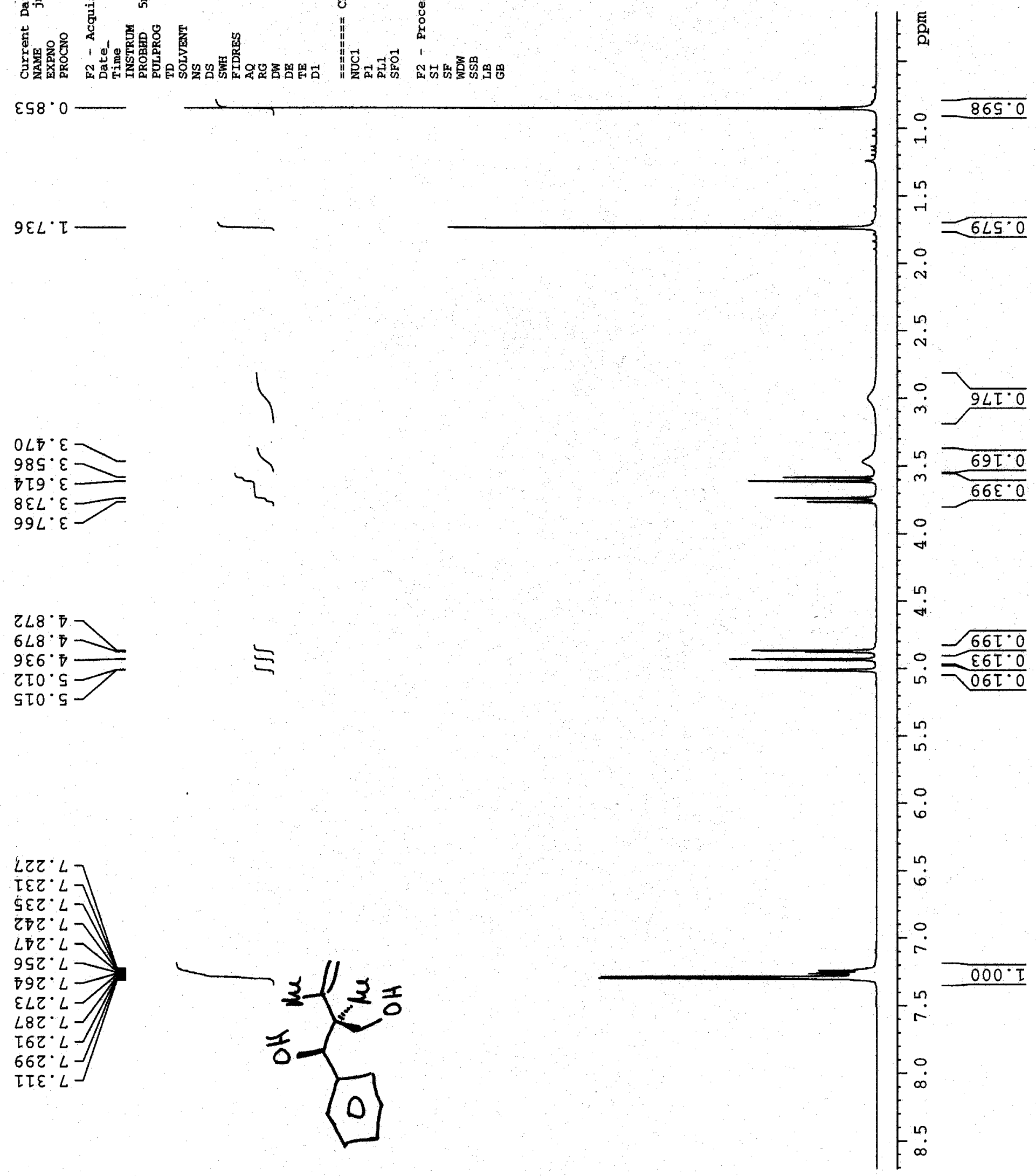


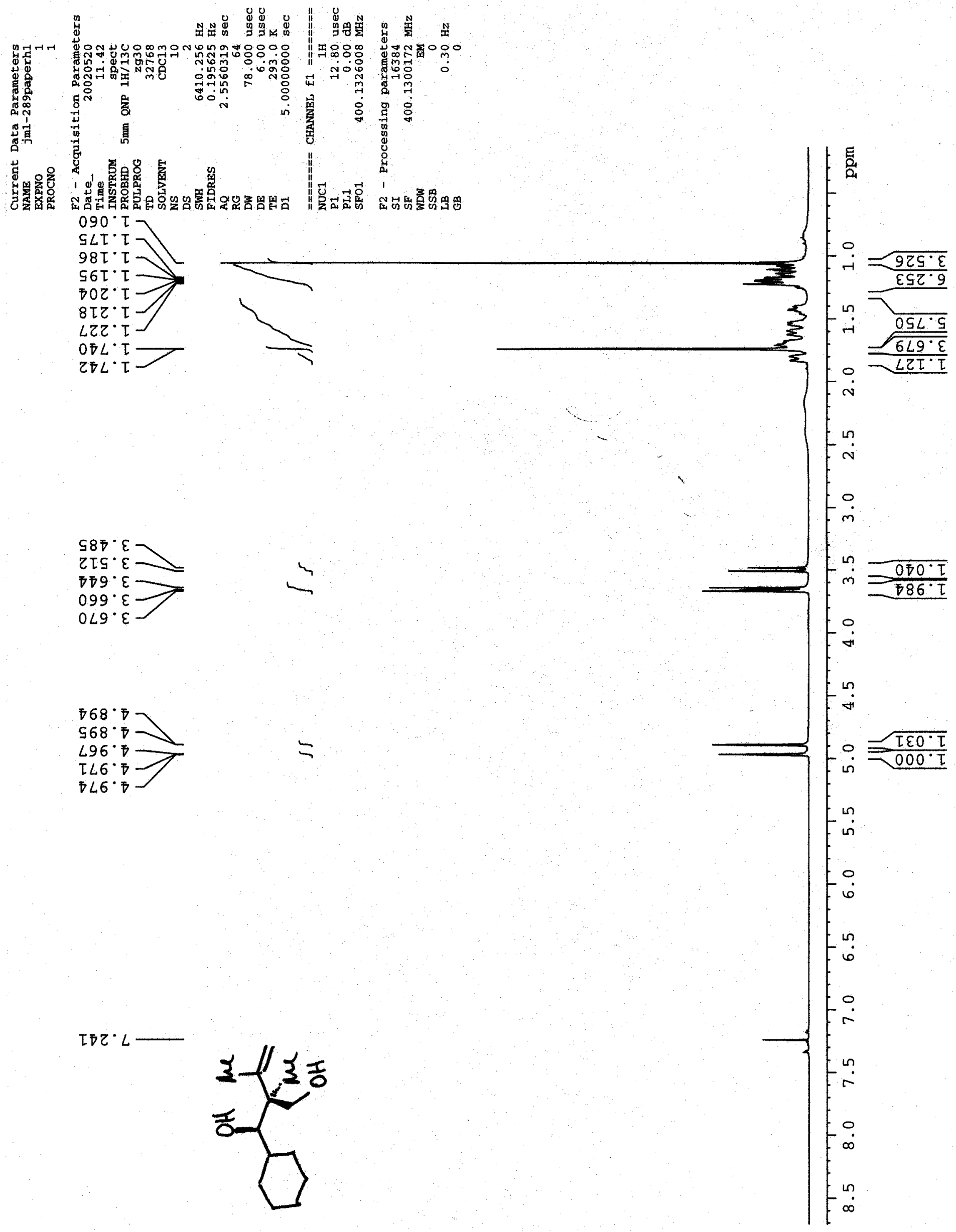




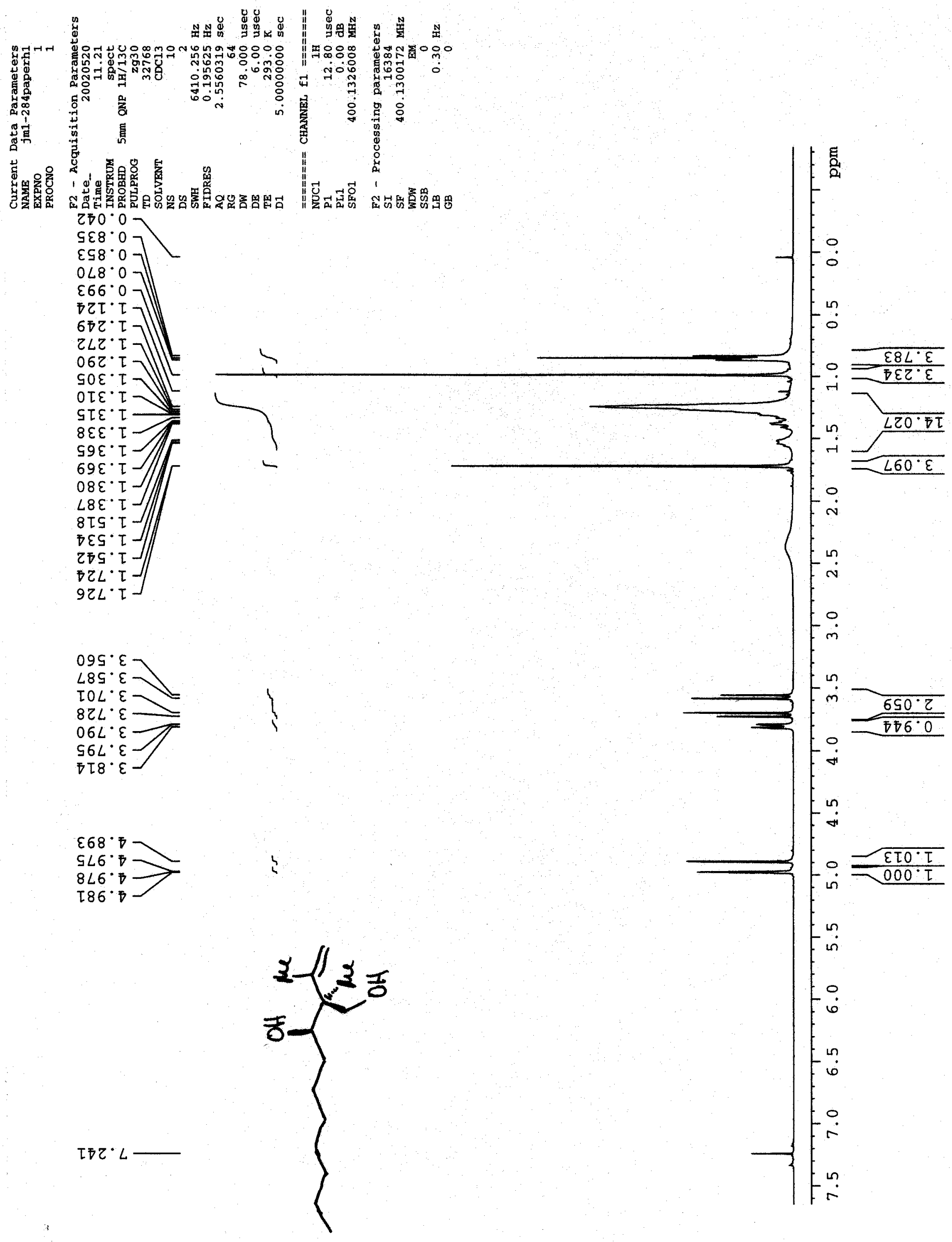




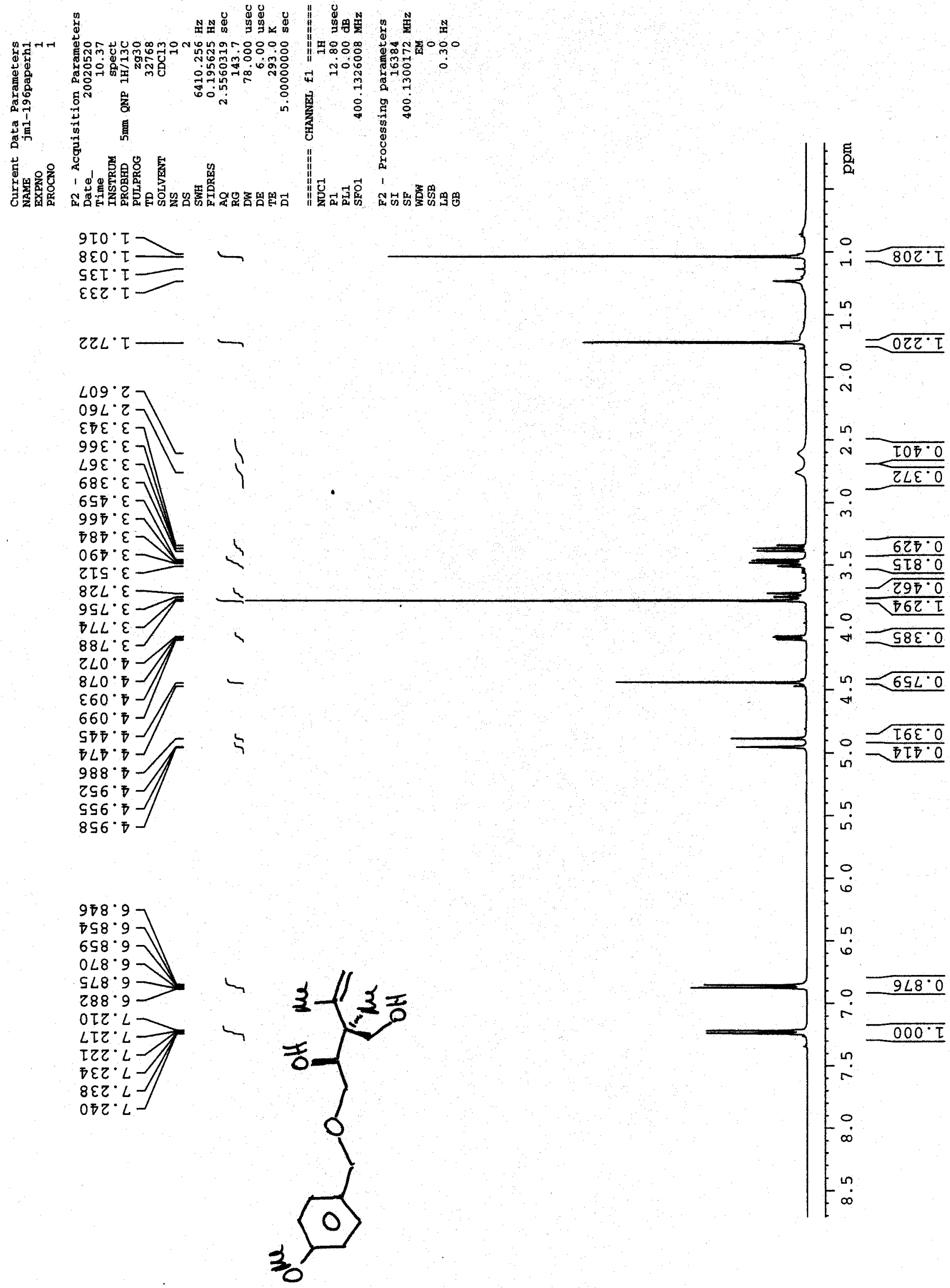




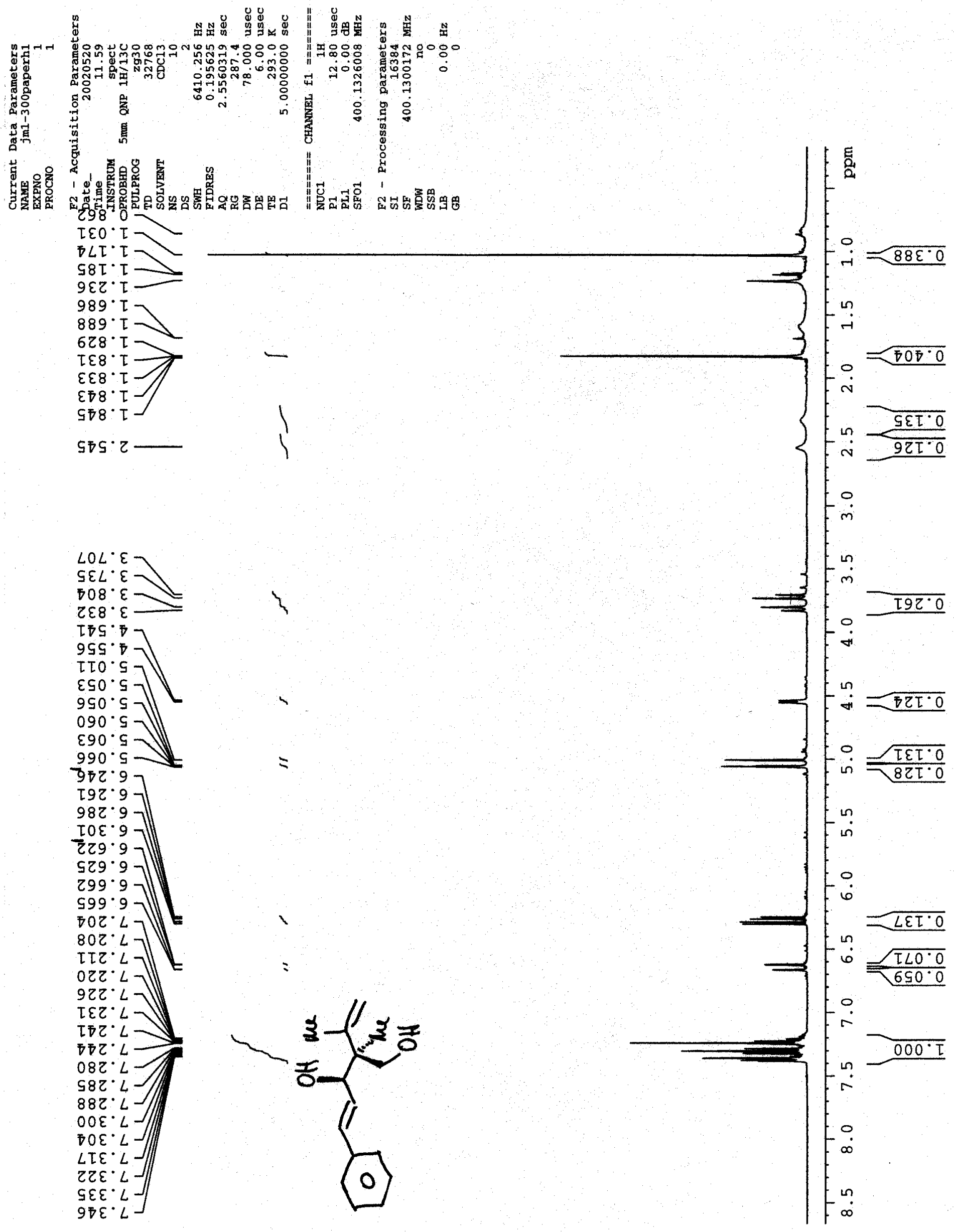




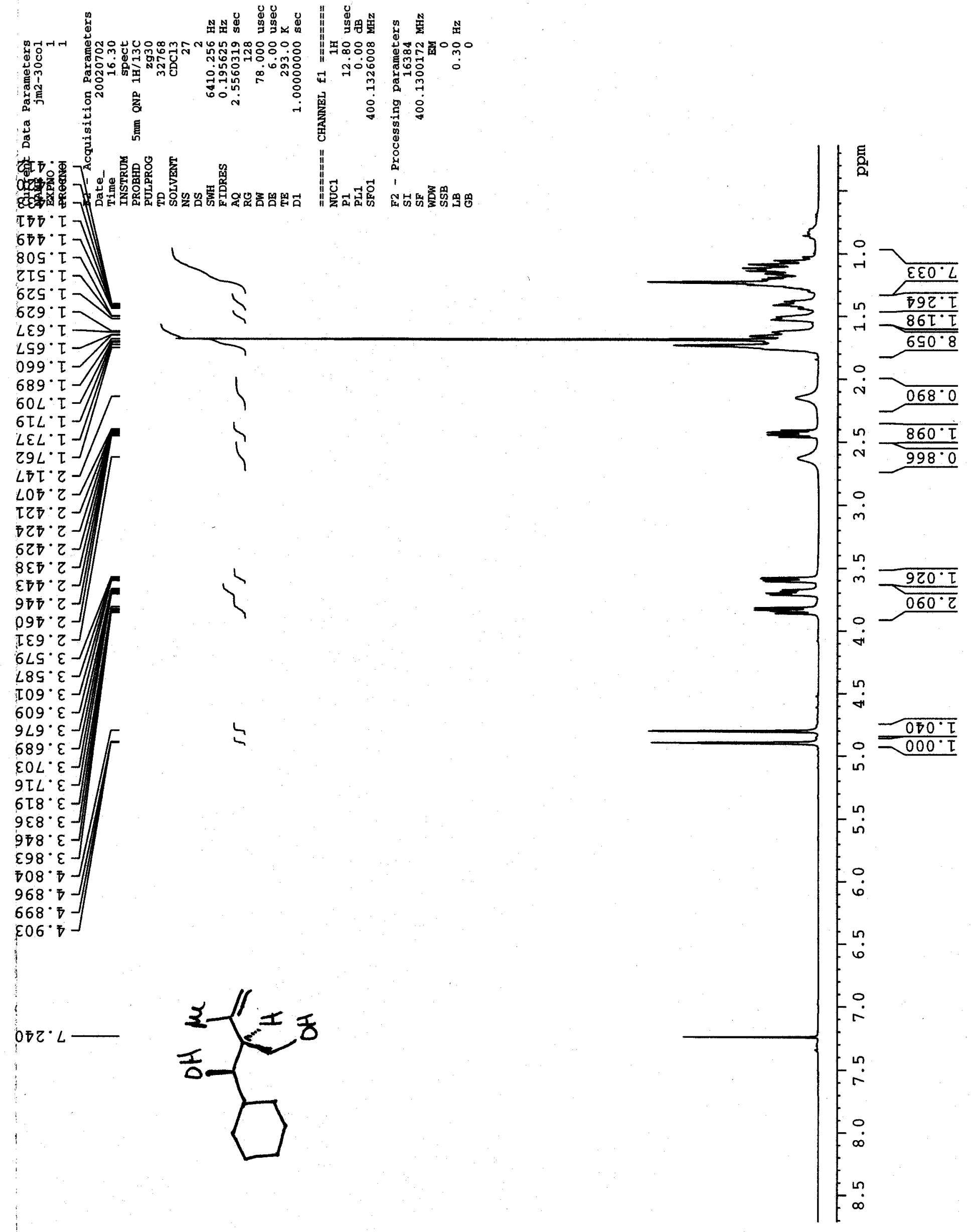




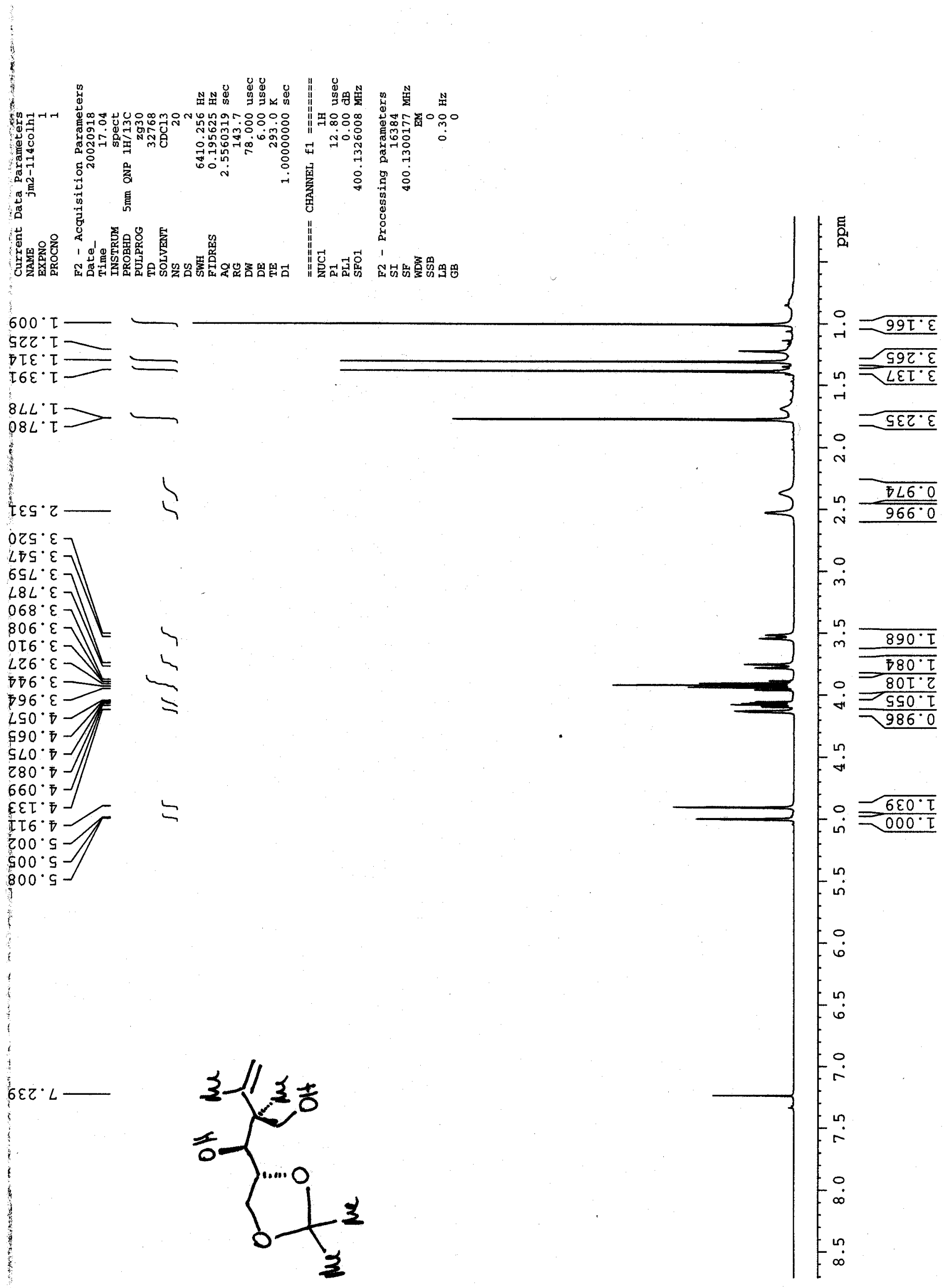




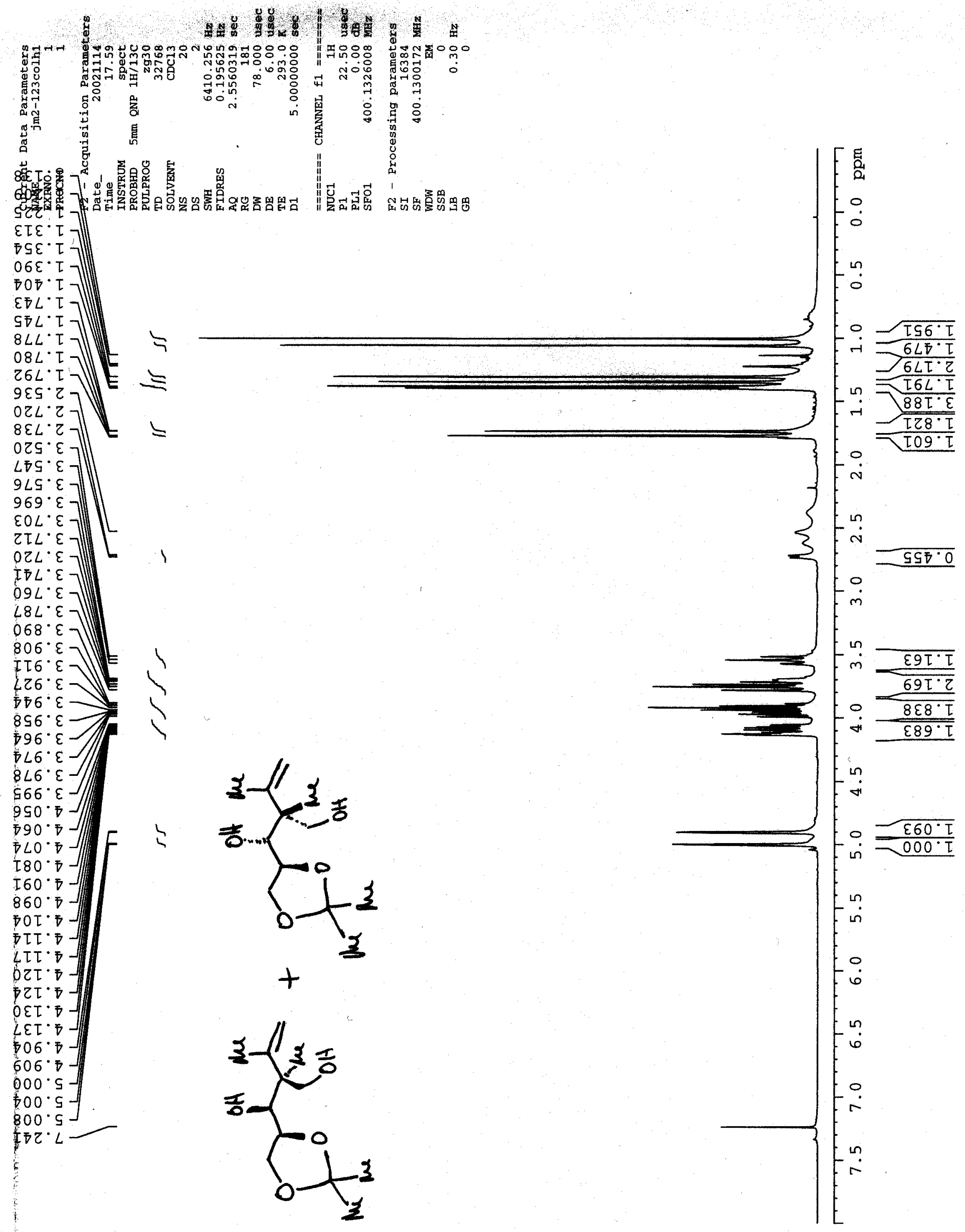

